\title{
Erratum to: Human immunodeficiency virus-related retinal microangiopathy and systemic cytomegalovirus disease association
}

Yuko Iwasaki • Narumichi Yamamoto - Tatsushi Kawaguchi • Noriko Ozaki $\cdot$ Makoto Tomita $\cdot$ Atsushi Ajisawa Manabu Mochizuki $\cdot$ Kimio Murakami

Published online: 20 August 2013

(C) Japanese Ophthalmological Society 2013

Erratum to: Jpn J Ophthalmol (2013) 57:372-378

DOI 10.1007/s10384-013-0247-7

The title in the PDF version of this article unfortunately contained an error. The space was missing between "retinal" and "microangiopathy", which should be written as two words. The corrected title is shown in this erratum.

The online version of the original article can be found under doi:10.1007/s10384-013-0247-7.

Y. Iwasaki $(\bowtie) \cdot N$. Yamamoto · T. Kawaguchi · N. Ozaki ·

K. Murakami

Department of Ophthalmology, Tokyo Metropolitan Komagome

Hospital, 18-22, Honkomagome 3chome, Bunkyo,

Tokyo 113-0021, Japan

e-mail: sahnya96@gmail.com

Y. Iwasaki · T. Kawaguchi · M. Mochizuki

Department of Ophthalmology and Visual Science,

Tokyo Medical and Dental University, Bunkyo, Japan

M. Tomita

Clinical Research Center, Tokyo Medical and Dental University,

Bunkyo, Japan

A. Ajisawa

Department of Infectious Diseases, Tokyo Metropolitan

Komagome Hospital, Bunkyo, Japan 